\title{
Síndrome inflamatorio multisistémico asociado a COVID-19 en niños y adolescentes
}

\author{
Multisystemic inflammatory syndrome associated with \\ COVID-19 in children and adolescents
}

\author{
Raymundo Rodríguez-Herrera,* Mauricio Rivera-Díaz ${ }^{\ddagger}$ \\ * Médico Pediatra adscrito al Departamento de Medicina Interna del Instituto Nacional de Pediatría. \\ Red de Enfermedad de Kawasaki en América Latina (REKAMLATINA). \\ ₹ Médico Familiar adscrito al Servicio de Urgencias del Hospital Pediátrico de Iztacalco.
}

A finales de diciembre de 2019 en Wuhan, China, se identificaron los primeros casos de una nueva enfermedad que produce síntomas similares a los de la gripe, entre los que se incluyen fiebre, tos, cefalea, disnea, mialgia y fatiga. En los casos graves provoca neumonía, síndrome de dificultad respiratoria aguda, sepsis y choque séptico; lo que conduce a la muerte al $3 \%$ de los adultos infectados. ${ }^{1,2}$

El 7 de enero de 2020 los científicos chinos aislaron el virus causante de la enfermedad como un nuevo coronavirus (2019-nCoV), posteriormente denominado el virus SARS-CoV- $2,{ }^{3}$ agente causal de la pandemia actual COVID-19 declarada como tal por la Organización Mundial de la Salud (OMS) el 11 de marzo de 2020. ${ }^{4}$ El acrónimo COVID-19 es el nombre designado del inglés coronavirus disease 2019 a una enfermedad infecciosa emergente que no había sido identificada previamente, contra la cual los humanos no tienen inmunidad preexistente. ${ }^{5}$

Desde el inicio de la pandemia por COVID-19, la experiencia en China y en otros países de Asia y Europa coincidían en que los pacientes menores de 18 años representaban el $2.4 \%$ de todos los casos reportados. La mayor parte de los pacientes eran asintomáticos o tenían una enfermedad leve, por lo que la mayoría de los niños infectados se

Financiamiento: Ninguno.

Conflicto de intereses: Ninguno.

https://dx.doi.org/10.35366/95645

Rev Latin Infect Pediatr 2020; 33 (3): 115-118 recuperaban una o dos semanas después de la aparición de los síntomas. ${ }^{1}$ De aquéllos que requerían hospitalización en salas de emergencia, salas de pediatría o unidades de cuidados intensivos pediátricos (UCIP), la mayoría se debían a dificultad respiratoria y neumonía. ${ }^{6}$

Hasta febrero de $2020^{7}$ no se habían informado muertes por la infección por coronavirus en el grupo de edad de cero a nueve años y en el grupo de 10 a 19 años la tasa de mortalidad fue de $0.2 \%$; sin embargo, en el grupo de edad de 70 a 79 y mayores de 80 años las tasas de mortalidad fueron de 8.0 y $14.8 \%$, respectivamente.

No obstante, durante la última semana de abril de 2020, se emitieron alertas por el Grupo de Estudio de Reumatología de la Sociedad Italiana de Pediatría, el Colegio Real de Pediatría y Salud del Niño, ${ }^{8}$ la Sociedad de Cuidados Intensivos Pediátricos del Reino Unido y la Asociación Española de Pediatría por el incremento de casos de enfermedad de Kawasaki (EK) incompletos o atípicos con mayor resistencia a la gammaglobulina intravenosa (GGIV), tendencia hacia el síndrome de activación macrofágica (SAM), estado de choque hiperinflamatorio y necesidad de ingreso a UCIP. ${ }^{8,9}$ En las primeras semanas de mayo de 2020 se empezaron a notificar casos semejantes en los Estados Unidos de América (EUA), especialmente en Nueva York, ${ }^{10}$ donde en pocas semanas el número superó los 200 casos.

Esta presentación grave en pacientes con COVID19 ha recibido varios nombres: síndrome pediátrico multisistémico inflamatorio, ${ }^{11}$ «Kawashocky», 
Rev Latin Infect Pediatr 2020; 33 (3): 115-118

«Coronasacki» o choque hiperinflamatorio en niños con COVID-19, síndrome inflamatorio pediátrico multisistémico asociado temporal a SARS-CoV-2 (PIMS-TS), y síndrome multisistémico inflamatorio en niños. ${ }^{12,13}$ Después de esta definición, el Centro para el Control y Prevención de Enfermedades de EUA (CDC) emitió el 14 de mayo una definición modificada de caso y modificó el término a MIS-C. ${ }^{14}$ Finalmente, la OMS define a esta nueva entidad con el nombre de síndrome inflamatorio multisistémico (SIM/MIS) en niños y adolescentes con COVID-19. ${ }^{15}$ Esta definición primaria de caso aplica para niños y adolescentes de cero a 19 años de edad que cumplan con los criterios de fiebre mayor de 3 días, y dos de los siguientes:

- Conjuntivitis bilateral no supurativa o signos de inflamación mucocutánea (boca, manos o pies).

- Hipotensión arterial o choque.

- Hallazgos de disfunción miocárdica, pericárdica, valvular o anormalidades coronarias (incluyendo hallazgos ecocardiográficos o aumento de troponina/NT-pro BNP).

- Evidencia de coagulopatía (por TP, TPT, elevación de dímeros $\mathrm{D}$ ).

- Manifestaciones gastrointestinales agudas (diarrea, vómitos o dolor abdominal).

- Elevación de marcadores de inflamación como velocidad de eritrosedimentación (VSG), proteína C-reactiva (PCR), o procalcitonina.

- Ausencia de otra causa de inflamación como sepsis bacteriana, síndrome de choque tóxico estafilocócico o estreptocócico.

- Evidencia de COVID-19 (RT-PCR, prueba de antígeno o serología positiva), o contacto probable con pacientes con COVID-19.

La primera publicación de casos pediátricos de SIM fue de Inglaterra el 6 de mayo de 2020. En este reporte,${ }^{13}$ los autores describen un aumento de casos en un lapso de 10 días a mediados de abril con las denominaciones de choque hiperinflamatorio, Kawasaki atípico, síndrome de choque por enfermedad de Kawasaki y síndrome de choque tóxico. A diferencia de la mayoría de niños con EK, todos ellos eran mayores de cinco años de edad, excepto un niño de cuatro años. Estos pacientes tuvieron predominio de síntomas gastrointestinales, todos progresaron a choque caliente vasopléjico refractario a volumen y requirieron de soporte inotrópico. Entre otros hallazgos, en la ecocardiografía un paciente presentó dilatación coronaria importante y otro, hiperrefringencia pericoronaria. Todos fueron tratados con GGIV y antimicrobianos, y siete de los ocho egresaron de la UCIP entre el día cuatro y seis. Un paciente de 14 años falleció debido a arritmia, choque refractario y accidente cerebrovascular. El SARS-CoV-2 fue confirmado en dos pacientes y en otros cuatro hubo contacto con familiares positivos.

La primera publicación de Italia al respecto proviene de investigadores del Hospital General de Bergamo, ${ }^{16}$ en la cual los autores comparan retrospectivamente dos periodos de tiempo de casos pediátricos semejantes a enfermedad de Kawasaki («Kawasaki-like»), del 1 de enero de 2015 al 17 de febrero de 2020, y del 18 de febrero al 20 de abril de 2020 . En este segundo periodo, ellos documentan 10 niños con cuadros de "Kawasaki-like» y de los cuales la mitad tuvieron presentación clínica clásica. Esto representó un incremento de 30 veces el número de casos respecto al periodo anterior. Comparativamente las edades de ambos grupos fueron tres (2-5 años) versus siete niños (3-5 años), y solamente uno de los 10 era menor de cinco años. En todos los pacientes, el aumento de marcadores de inflamación aguda fue común, pero todos los hemocultivos fueron estériles. La RT-PCR para SARS-CoV-2 fue positiva en dos de ellos, la lgG positiva en $80 \%$, mientras que la IgM sólo en $30 \%$. El ecocardiograma fue anormal en seis (60\%) de ellos y dos pacientes tuvieron aneurismas coronarios mayores de $4 \mathrm{~mm}$. Todos los pacientes fueron tratados con GGIV, metilprednisolona como terapia coadyuvante en ocho de ellos, ácido acetilsalicílico (AAS) en dos, e inotrópicos en dos. Todos respondieron al tratamiento, fueron egresados y están en vigilancia clínica y ecocardiográfica.

Belhadjer $Z$ y colegas, ${ }^{17}$ en una publicación reciente, analizaron retrospectivamente 35 niños que se hospitalizaron con disfunción sistólica aguda ventricular izquierda o choque cardiogénico y estado de inflamación multisistémica en 12 hospitales de Francia y uno de Suiza entre el 22 de marzo y el 30 de abril de 2020. La mediana de edad fue de 10 años, ninguno tenía cardiopatía de fondo. Se documentó infección por SARS-CoV-2 en 31 (88.5\%) pacientes e historia de contacto reciente con familiares con cuadro viral respiratorio agudo en $37 \%$. Ochenta por ciento tuvo síntomas gastrointestinales y dos de ellos requirieron incluso laparotomía exploratoria por abdomen agudo. Ochenta y tres por ciento fueron admitidos directamente a la UCIP, $80 \%$ de los pacientes estaban en choque cardiogénico y requirieron fármacos inotrópicos. El ecocardiograma documentó dilatación de las arterias 
coronarias en $17 \%$, pero no aneurismas en el primer ecocardiograma. No hubo fallecidos.

Whittaker y colaboradores, en una serie de 58 niños hospitalizados que cumplieron con la definición de trastornos inflamatorios multisistémicos infantiles propuesto recientemente en el Reino Unido, Estados Unidos, o por la OMS, tuvieron un amplio espectro de presentación de signos y síntomas, incluyendo fiebre, síntomas gastrointestinales, y erupción cutánea, así como la gravedad de la enfermedad, incluida lesión del miocardio, choque y desarrollo de aneurismas de las arterias coronarias. Los casos reportados en este estudio proporcionan evidencia de un espectro más amplio de enfermedad que el identificado en la definición inicial del Reino Unido y los primeros informes. Además, provisionalmente parecen existir tres patrones de enfermedad entre los niños hospitalizados con PIMS-TS. Un grupo de niños tenía fiebre persistente y niveles elevados de marcadores inflamatorios, pero sin características de EK, choque o insuficiencia orgánica. Un segundo grupo cumplió los criterios de diagnóstico para EK y un tercer grupo tuvo choque y evidencia clínica, ecocardiográfica y de laboratorio de lesión miocárdica. La comparación con pacientes de cohortes con EK, síndrome de choque en EK y síndrome de choque tóxico proporciona más información sobre este síndrome y sugiere que PIMS-TS difiere de estas entidades inflamatorias pediátricas. Las características clínicas y de laboratorio de estos grupos pueden proporcionar información útil para el nuevo síndrome. ${ }^{18}$

Con base en las publicaciones analizadas anteriormente este particular síndrome comparte algunas características con otros procesos inflamatorios pediátricos: enfermedad de Kawasaki atípica o incompleta, síndrome de choque tóxico (estreptococo y estafilococo), sepsis bacteriana y los síndromes de activación de macrófagos; sin embargo también tiene algunas diferencias que se comentan a continuación.

A diferencia de los casos de Kawasaki clásico, que en $90 \%$ de ellos ocurre en niños menores de cinco años de edad, en el informe de casos de SIM suelen ser escolares o adolescentes. Afecta principalmente a personas de ascendencia afroamericana, caribeña e hispana, mientras que la EK afecta principalmente a los de ascendencia asiática-oriental.

Los pacientes tienen fiebre persistente, elevada y de difícil control con datos clínicos de EK incompleta. Tienen manifestaciones gastrointestinales agudas con un cuadro de dolor abdominal intenso que incluso requirió de laparotomía exploradora en algunos casos. La mayoría no tuvo afectación respiratoria significativa, aunque recibieron ventilación mecánica como parte de la estabilización cardiovascular. Todos evolucionaron a choque caliente, resistente a la resucitación con volumen; se requirió la administración de aminas para alcanzar una respuesta hemodinámica. Algunos niños tuvieron edema pleural, pericárdico o ascitis, sugerente de proceso inflamatorio. La insuficiencia cardiaca, al parecer, se debe a edema miocárdico, más que a daño miocárdico inflamatorio. Se acompaña de neutrofilia, linfopenia y alteración en los marcadores de la inflamación: elevación de proteína $C$ reactiva, elevación de dímero $D$, fibrinógeno, procalcitonina, ferritina, disfunción multiorgánica (cardiovascular, respiratoria, renal, hepática, gastrointestinal, hematológica y neurológica).

Tienen inflamación más intensa y niveles más elevados de marcadores cardiacos de lesión que EK. La prueba de PCR en tiempo real para diagnóstico de SARS-CoV-2 puede ser positiva o negativa. La ausencia de síntomas anteriores de COVID-19, y a menudo un resultado negativo de reacción en cadena de la polimerasa, a veces anticuerpos positivos o exposición familiar, y el desarrollo de SIM después de un retraso de tres a seis semanas sugiere que el SARS-CoV-2 puede estar actuando como desencadenante, lo que indica que se trata de dos entidades separadas y que el tratamiento para SIM puede ser diferente que el de la EK. Su manifestación inicial es muy severa, donde media una tormenta de citoquinas similar a la descrita en adultos, pero con diferente espectro clíni$\mathrm{co}$, afortunadamente responden a la administración de gammaglobulina y esteroide la mayoría de los pacientes. El aumento de marcadores de inflamación aguda incluidos PCR, ferritina, troponina, dímero $D$ y los niveles de proBNP y NT-proBNP pueden ser útiles para predecir la progresión de la enfermedad.

El diagnóstico temprano del síndrome inflamatorio multisistémico en niños y adolescentes con COVID19 complicada por insuficiencia cardiaca es muy útil en el periodo actual para identificar a los niños que requieren tratamiento y para prevenir la disfunción ventricular izquierda y la insuficiencia cardiaca aguda. Belhadjer Z y su equipo proponen un algoritmo simple en la sala de urgencias para niños con fiebre prolongada e inexplicable, incluyendo una rápida determinación en sangre de la fracción $\mathrm{N}$-terminal del propéptido natriurético de tipo $\mathrm{B}$ (NT-proBNP) para una evaluación especializada urgente en aquellos pacientes con niveles elevados de NT-proBNP, y un manejo más clásico para aquéllos sin evidencia de compromiso cardiaco temprano. Con este protocolo 
Belhadjer Z y su grupo observaron un aumento en el número de pacientes con formas menos graves de la enfermedad sin insuficiencia cardiaca y un número estable de ingresos por insuficiencia cardiaca sin necesidad de asistencia mecánica.

Un denominador común en las series señaladas anteriormente es que la mayoría de los pacientes recibió tratamiento con GGIV con y sin esteroides. El uso de anakinra, un antagonista del receptor de la IL-1, rara vez se indicó. Este fármaco actúa bloqueando la tormenta de citocinas que, obviamente, juega un papel en esta condición y podría ser un tratamiento alternativo en formas resistentes. Otro posible tratamiento es el tocilizumab, medicamento dirigido contra el receptor de la interleucina-6.

El diagnóstico y el tratamiento temprano parece conducir a resultados favorables usando terapias clásicas. Todavía no está claro si se trata de una complicación postinfecciosa o una complicación primaria de la infección con SARS-CoV-2; sin embargo, las descripciones epidemiológicas iniciales son altamente sugestivas de una correlación. Se desconoce cuál es el mejor tratamiento porque se requiere un mayor número de casos y ensayos clínicos controlados.

Por lo anterior, las comunidades pediátricas y de cardiología deben estar muy alerta de la existencia de esta nueva enfermedad probablemente relacionada con la infección por SARS-CoV-2, que comparte similitudes con la enfermedad de Kawasaki, pero que tiene un mayor riesgo de complicaciones relacionadas con inflamación multisistémica y el desarrollo de disfunción sistólica aguda ventricular izquierda, choque cardiogénico o síndrome de activación macrofágica.

\section{REFERENCIAS}

1. Report of the WHO-China Joint Mission on Coronavirus Disease 2019 (COVID-19). 2020. Disponible en: https:// www.who.int/docs/default-source/coronaviruse/who-chinajoint-missionon-covid-19-final-report.pdf

2. Zhang Y. The epidemiological characteristics of an outbreak of 2019 Novel Coronavirus Diseases (COVID19)-China, 2020. Chinese Journal of Epidemiology (by The Novel Coronavirus Pneumonia Emergency Response Epidemiology Team). 2020.

3. World Health Organization. Naming the coronavirus disease (COVID-19) and the virus that causes it. World Health Organization; 2020. Available in: www.who.int/emergencies/ diseases/novel-coronavirus-2019/

4. Noticias ONU. El coronavirus es una pandemia. Disponible en: https://news.un.org/es/story/2020/03/1470991

5. Organización Mundial de la Salud (OMS). Intervención del Director General de la OMS en la conferencia de prensa sobre el 2019-nCoV del 11 de febrero de 2020. 11 de febrero de 2020.
6. Liu W, Zhang Q, Chen J, Xiang R, Song H, Shu S et al. Detection of Covid-19 in children in early January 2020 in Wuhan, China. N Engl J Med. 2020; 382: 1370-1371.

7. Worldometer (2020). Age, sex, existing conditions of COVID-19 cases and deaths. February 29, 4:40 GMT [cited 2020 Mar 22]. Available in: https://www.worldometers.info/ coronavirus/coronavirus-age-sex-demographics/

8. Royal College of Paediatrics and Child Health. Guidance: paediatric multisystem inflammatory syndrome temporally associated with COVID-19. Accessed May 22, 2020. Available in: https://www.rcpch.ac.uk/resources/guidancepaediatric-multisystem-inflammatory-syndrome-temporallyassociated-covid-19-pims

9. Información para las familias respecto al comunicado interno: "Alerta sobre casos de shock pediátrico". Difundido en redes sociales. Disponible en: https://www.aeped.es/ noticias/informacion-las-familiasrespecto-al-comunicadointerno-alerta-sobrecasos-shock-pediatrico

10. 2020 Health Alert \#13: Pediatric multi-system inflammatory syndrome potentially associated with COVID-19. Available in: https://www1.nyc.gov/assets/doh/downloads/ pdf/han/ alert/2020/covid-19-pediatric-multi-systeminflammatorysyndrome.pdf

11. Ulloa GR, Ivankovich EG, Yamazaki NM. Síndrome inflamatorio multisistémico asociado a COVID-19 en niños y adolescentes: un Ilamado al diagnóstico. Rev Chilena Infectol. 2020; 37 (3): 199-201.

12. Hennon TR, Penque MD, Abdul-Aziz R et al. COVID-19 associated multisystem inflammatory syndrome in children (MIS-C) guidelines; a western New York approach [published online ahead of print, 2020 May 23]. Prog Pediatr Cardiol. 2020; 101232. doi: 10.1016/j.ppedcard.2020.101232.

13. Riphagen S, Gomez X, Gonzalez-Martinez C, Wilkinson $\mathrm{N}$, Theocharis $\mathrm{P}$. Hyperinflammatory shock in children during COVID-19 pandemic. Lancet. 2020. pii: S01406736(20)31094-1.

14. Centers for Disease Control and Prevention. Emergency preparedness and response: health alert network. Published May 14, 2020. Accessed May 22, 2020. Available in: https://emergency.cdc.gov/han/ 2020/han00432.asp

15. World Health Organization. Multisystem inflammatory syndrome in children and adolescents temporally related to COVID-19. 2020. Available in: https://www. who.int/news-room/commentaries/ detail/multisysteminflammatory-syndrome-in-children and-adolescents-withcovid-19

16. Verdoni L, Mazza A, Gervasoni A et al. An outbreak of severe Kawasaki-like disease at the Italian epicentre of the SARS-CoV-2 epidemic: an observational cohort study. Lancet. 2020; 395 (10239): 1771-1778.

17. Belhadjer Z, Méot M, Bajolle F et al. Acute heart failure in multisystem inflammatory syndrome in children (MIS-C) in the context of global SARS-CoV-2 pandemic. Circulation. 2020; 10.1161/CIRCULATIONAHA.120.048360.

18. Whittaker E, Bamford A, Kenny J et al. Clinical characteristics of 58 children with a pediatric inflammatory multisystem syndrome temporally associated with SARSCoV-2 [published online ahead of print, 2020 Jun 8]. JAMA. 2020; e2010369. doi: 10.1001/jama.2020.10369.

Correspondencia:

Raymundo Rodríguez-Herrera

E-mail: raymundo.rdzh@gmail.com 